\title{
Rastreamento do câncer do colo do útero em um estado do nordeste brasileiro
}

\author{
Trial of cervical cancer in a northeast brazilian state \\ Rastreo del cáncer del colo del útero en un estado del nordeste brasileño \\ Gleyson Moura dos SANTOS ${ }^{1}$ \\ Bruna Grazielle Mendes RODRIGUES ${ }^{2}$ \\ Geovana Chaves Ximenes de MORAIS ${ }^{2}$ \\ Rodolfo Vieira FONTENELE ${ }^{3}$ \\ Carolina Rodrigues de Oliveira SOUSA \\ Marilene Magalhães de BRITO ${ }^{5}$ \\ ${ }^{1}$ Nutricionista. Mestrando em Ciências e Saúde (PPGCS/UFPI). Pós-Graduando em Fitoterapia Aplicada à Nutrição (UCAM) 64085-300 Teresina-PI, Brasil
${ }^{2}$ Acadêmica do Curso de Nutrição (UFPI) 64048-480, Teresina-PI, Brasil
${ }^{3}$ Acadêmico do Curso de Medicina (UFPI) 64048-480, Teresina-PI, Brasil
${ }^{4}$ Enfermeira. Mestre em Saúde e Comunidade (PPGSC/UFPI). Pós-Graduada em Enfermagem do Trabalho (UNIPÓS)
${ }^{5}$ Nutricionista. Mestre em Alimentos e Nutrição (PPGAN/UFPI). Pós-graduanda em Nutrição Clínica e Esportiva (UCAM) 64048-480, Teresina -PI, Brasil
}

\section{Resumo}

Introdução: O câncer do colo do útero caracteriza-se como um importante problema de saúde pública mundial. Objetivo: Diante do exposto o presente artigo objetivou rastrear os casos de câncer de colo de útero em um estado do nordeste brasileiro. Material e Método: Trata-se de um estudo descritivo/retrospectivo observacional de base populacional, utilizando dados dos exames citopatológicos realizados entre os anos de 2012 a 2014 no estado do Piauí, registrados no Sistema de Informação do câncer do colo do útero (SISCOLO) e disponibilizados no site do Departamento de Informática do Sistema Único de Saúde (DATASUS). Resultados: Foram registradas no SISCOLO 513.910 citologias de mulheres residentes nos municípios do estado do Piauí. Observa-se um maior número de exames realizados do ano de 2013, com a maioria desses realizados em mulheres na faixa etária de 25 a 64 anos, pardas e com ensino fundamental incompleto. O epitélio escamoso foi o mais representado nas amostras citopatológicas, sendo os agentes microbiológicos mais frequentes no colo uterino os cocos, os Lactobacillus sp e os Bacilos. Foi verificado dentre as alterações celulares benignas a inflamação como achado mais presente e as células escamosas indeterminadas não neoplásicas foram as atipias em células escamosas mais presente. Conclusão: Dessa forma é importante a adoção de estratégias que melhorem a coleta desses dados, que são importantes fontes de informações em saúde e que podem ser utilizados para a implementação de possíveis políticas nesse campo e em pesquisas da área de saúde.

Descritores: Neoplasias do Colo do Útero; Programas de Rastreamento; Saúde Pública.

\begin{abstract}
Introduction: Cervical cancer is characterized as a major public health problem worldwide. Objective: In view of the above, this article aimed to track the cases of cervical cancer in a state in the Brazilian Northeast. Methods: This is a population-based observational descriptive / retrospective study using data from the cytopathological examinations performed between the years of 2012 to 2014 in the state of Piauí, registered in the Cervical Cancer Information System (SISCOLO) and made available on the website of the Department of Informatics of the Unified Health System (DATASUS). Results: 513,910 Pap smears were registered in SISCOLO of women living in the municipalities of the state of Piauí. A greater number of examinations were carried out in the year 2013, with the majority of the exams being carried out in women between the ages of 25 and 64 years, with browns and incomplete primary education. The squamous epithelium was the most represented in the cytopathological samples, being the microbiological agents more frequent in the cervix the cocci, the Lactobacillus sp and the Bacilli. Among the benign cellular alterations the inflammation was found as the most present finding and the undetermined squamous non-neoplastic cells were the most present squamous cell atypia. Conclusion: Thus, it is important to adopt strategies that improve the collection of these data, which are important sources of health information and can be used to implement possible policies in this field and in health research.
\end{abstract}

Descriptors: Uterine Cervical Neoplasms; Mass Screening; Public Health.

\section{Resumen}

Introducción: El cáncer de cuello de útero se caracteriza como un importante problema de salud pública mundial. Objetivo: Ante el expuesto el presente artículo objetivó rastrear los casos de cáncer de cuello de útero en un estado del nordeste brasileño. Métodos: Se trata de un estudio descriptivo / retrospectivo observacional de base poblacional, utilizando datos de los exámenes citopatológicos realizados entre los años de 2012 a 2014 en el estado de Piauí, registrados en el Sistema de Información del cáncer del cuello del útero (SISCOLO) y disponibles en el sitio del Departamento de Informática del Sistema Único de Salud (DATASUS). Resultados: Se registraron en el SISCOLO 513.910 citologías de mujeres residentes en los municipios del estado de Piauí. Se observa un mayor número de exámenes realizados del año 2013 , con la mayoría de estos realizados en mujeres en el grupo de edad de 25 a 64 años, pardas y con enseñanza fundamental incompleta. El epitelio escamoso fue el más representado en las muestras citopatológicas, siendo los agentes microbiológicos más frecuentes en el cuello uterino los cocos, los Lactobacillus sp y los Bacilos. Se verificó entre las alteraciones celulares benignas la inflamación como hallazgo más presente y las células escamosas indeterminadas no neoplásicas fueron las atipias en células escamosas más presentes. Conclusión: De esta forma es importante la adopción de estrategias que mejoren la recolección de esos datos, que son importantes fuentes de información en salud y que pueden ser utilizados para la implementación de posibles políticas en ese campo y en investigaciones del área de salud.

Descriptores: Neoplasias del Cuello Uterino; Tamizaje Masivo; Salud Publica.

\section{INTRODUÇÃO}

O câncer do colo do útero caracteriza-se como um importante problema de saúde pública mundial ${ }^{1}$. Essa neoplasia está associada à infecção persistente por subtipos oncogênicos do vírus HPV (papilomavírus humano), especialmente o HPV-16 e o HPV-18, responsáveis por cerca de 70\% dos cânceres cervicais ${ }^{2}$.

Segundo dados do Instituto Nacional de Câncer (INCA), para o Brasil, estimam-se 16.370 novos casos de câncer do colo do útero para cada ano do biênio 2018-2019, com um risco estimado de 15,43 casos a cada 100 mil mulheres. Sem considerar os tumores de pele não melanoma, o câncer do colo do útero é o segundo mais incidente na região Nordeste $(20,47 / 100 \mathrm{mil})^{3}$.

Essa neoplasia apresenta evolução lenta, passando por fases pré-clínicas detectáveis e curáveis, tem início na forma de uma lesão 
precursora, que pode ou não evoluir para um processo invasivo no decorrer de um período de 10 a $20 \operatorname{anos}^{4}$. Dentre todos os tipos de câncer, é o que apresenta um dos mais altos potenciais de prevenção e cura. Sua maior incidência situa-se entre mulheres de 40 a 60 anos de idade, e apenas uma pequena porcentagem, naquelas com menos de 30 anos 5 .

Segundo a Organização mundial de Saúde um rastreamento, que realize uma cobertura da população alvo em torno de $80 \%$, através da colpocitologia oncótica, pode gerar uma redução em média de $60 \%$ a $90 \%$ na incidência do câncer do colo do útero ${ }^{6}$.

Porém, em estudo de Silva e Hortale ${ }^{7}$, foi observado que no Brasil, apenas 15\% da população feminina acima de 20 anos realiza esse exame, mesmo considerando um percentual de mulheres que utilizam serviços não públicos.

Sabe-se que a prevenção primária do câncer do colo do útero está diretamente relacionada à diminuição do risco de contágio pelo HPV. A transmissão desse vírus ocorre por via sexual, presumidamente através de abrasões microscópicas na mucosa ou na pele da região anogenital ${ }^{8}$.

Dessa forma e pelos elevados índices de incidência e mortalidade por câncer do colo do útero no Brasil é que se justifica a implantação de estratégias efetivas de controle dessas doenças que incluam ações de promoção à saúde, prevenção e detecção precoce e tratamento?.

Diante do exposto o presente artigo objetivou rastrear os casos de câncer de colo de útero em um estado do nordeste brasileiro.

\section{MATERIAL E MÉTODO}

Trata-se de um estudo
descritivo/retrospectivo observacional de base populacional, utilizando dados dos exames citopatológicos realizados entre os anos de 2012 a 2014 no estado do Piauí, registrados no Sistema de Informação do câncer do colo do útero (SISCOLO) e disponibilizados no site do Departamento de Informática do Sistema Único de Saúde (DATASUS).

Foram selecionadas as variáveis referentes à idade, grau de escolaridade, raça/cor, adequabilidade do material coletado, epitélios representados, microbiologia e alterações celulares. As idades foram categorizadas em faixas etárias pré-estabelecidas (abaixo de 25; 25 a 64 e 65 ou mais anos), priorizando- se a faixa de 25 a 64 anos conforme recomendação do Ministério da Saúde ${ }^{8}$.

A adequabilidade do material celular foi classificada como satisfatória ou não ${ }^{1}$. Os epitélios representados foram identificados no laudo dos exames como escamoso, glandular e/ou metaplásico. Os agentes microbiológicos, alterações celulares benignas, atipias em células escamosas e atipias em células glandulares também foram determinados.

Para tabulação e análise dos dados mediante estatísticas descritivas foram utilizados os programas Tabwin versão 3.6 e o Microsoft Office Excel 2010.

Por se tratar de uma análise fundamentada em banco de dados secundários e de domínio público, o estudo não foi encaminhado para apreciação de Comitê de Ética em Pesquisa, mas ressalta-se que foram tomados os cuidados éticos que preceituam a Resolução 466/12, do Conselho Nacional de Saúde ${ }^{10}$.

RESULTADOS

No período de 2012 a 2014, foram registradas no SISCOLO 513.910 citologias de mulheres residentes nos municípios do estado do Piauí. Desse total de exames, o ano de 2013, foi o que mais se destacou, apresentando $39,2 \%$ dos registros de citologias realizadas (Tabela 1).

Tabela 1. Distribuição de exames citopatológicos do colo do útero realizados por ano no estado do Piauí, no período de 2012 a 2014

\begin{tabular}{cc}
\hline Ano & $\begin{array}{c}\text { Quantidade de Exames } \\
\text { Citopatológicos }\end{array}$ \\
\hline 2012 & 169.330 \\
2013 & 201.318 \\
2014 & 143.262 \\
\hline Total & 513.910 \\
\hline Fonte: Ministério da Saúde \\
SISCOLO- Sistema de Informação do câncer do colo do útero
\end{tabular}

Do total de exames, $77,2 \%$ foram realizados para mulheres na faixa etária de 25 a 64 anos. O ensino fundamental incompleto foi o nível de escolaridade mais prevalente $(10,6 \%)$, entretanto esta variável foi ignorada ou não preenchida em $77,7 \%$ dos exames, mesmo fato observado para variável raça/cor, onde prevaleceram à cor parda (12\%) e $85,6 \%$ dos exames estavam sem esta informação (Tabela 2).

Tabela 2. Caracterização das variáveis demográficas da população estudada no estado do Piauí, no período de 2012 a 2014

\begin{tabular}{ccc}
\hline Variáveis & $\mathbf{N}$ & $\mathbf{\%}$ \\
\hline Faixa Etária & & \\
\hline$<25$ & 89.780 & 17,5 \\
$25-64$ & 396.904 & 77,2 \\
$>64$ & 27.226 & 5,3 \\
\hline Escolaridade & & \\
\hline Ignorado/em branco & 399.145 & 77,7 \\
Analfabeta & 10.678 & 2,1 \\
Ensino Fundamental Incompleto & 54.298 & 10,6 \\
Ensino Fundamental Completo & 20.458 & 3,9 \\
Ensino Médio Completo & 24.100 & 4,7 \\
Ensino Superior Completo & 5.231 & 1,0 \\
\hline Raça/cor & & \\
\hline Branca & 7.823 & 1,5 \\
Preta & 4.221 & 0,87 \\
Parda & 61.791 & 12,0 \\
Amarela & 78 & 0,028 \\
Indígena & 06 & 0,002 \\
Sem Informação & 439.991 & 85,6 \\
\hline
\end{tabular}

Fonte: Ministério da Saúde/SISCOLO- Sistema de Informação do câncer do colo do útero 
Considerando-se a adequabilidade do material celular, 92,8\% apresentaram celularidade adequada, sendo consideradas satisfatórias. Conforme a Tabela 3, o epitélio escamoso foi o mais representado nas amostras citopatológicas $(98,9 \%)$, seguido pelo glandular $(48,25 \%)$.

Tabela 3. Epitélios representados na amostra citopatológica da população estudada no estado do Piauí, no período de 2012 a 2014

\begin{tabular}{ccc}
\hline Epitélios & $\mathbf{N}$ & $\mathbf{\%}$ \\
\hline Escamoso & 508.249 & 98,90 \\
Glandular & 247.965 & 48,25 \\
Metaplásico & 66.872 & 13,01 \\
\cline { 2 - 3 } Fonte: Ministério da Saúde & &
\end{tabular}

SISCOLO- Sistema de Informação do câncer do colo do útero

Os agentes microbiológicos mais frequentes no colo uterino foram os cocos $(67,9 \%)$, os Lactobacillus sp $(35,7 \%)$ e os Bacilos $(25,6 \%)$ (Tabela 4).

Tabela 4. Principais agentes microbiológicos ao exame citopatológico da população estudada no estado do Piauí, no período de 2012 a 2014

\begin{tabular}{ccc}
\hline Microbiologia & $\mathbf{N}$ & $\mathbf{\%}$ \\
\hline Lactobacillus $s p$ & 183.447 & 35,7 \\
Cocos & 348.812 & 67,9 \\
Chlamydia sp & 64 & 0,01 \\
Actinomyces sp & 16 & 0,003 \\
Trichomonas vaginalis & 9.106 & 1,8 \\
Efeitos citopáticos compatível com & 46 & 0,009 \\
vírus do grupo Herpes & & \\
Bacilos & 131.598 & 25,6 \\
Outros & 32.693 & 6,4 \\
\hline
\end{tabular}

Fonte: Ministério da Saúde/SISCOLO- Sistema de Informação do câncer do colo do útero

Dentre as alterações celulares benignas (reativas ou reparativas), a inflamação foi o achado mais frequente $(77,3 \%)$. As células escamosas indeterminadas não neoplásicas foram as atipias em células escamosas mais frequentes, presente em 4.445 exames $(0,9 \%)$. O carcinoma epidermóide invasor foi evidenciado em $0,002 \%$ e adenocarcinoma in situ e invasor em $0,001 \%$ e $0,002 \%$ respectivamente (Tabela 5 ).

\begin{tabular}{|c|c|c|}
\hline Variáveis & $\mathbf{N}$ & $\%$ \\
\hline \multicolumn{3}{|l|}{ Alterações celulares benignas } \\
\hline Inflamação & 397.429 & 77,3 \\
\hline Atrofia & 49.553 & 9,6 \\
\hline Metaplasia & 22.733 & 4,4 \\
\hline Radiação & 492 & 0,1 \\
\hline Reparação & 7.064 & 1,4 \\
\hline Outros & 135.524 & 26,4 \\
\hline \multicolumn{3}{|l|}{ Atipias Celulares } \\
\hline $\begin{array}{l}\text { Células escamosas indeterminadas não } \\
\text { neoplásicas }\end{array}$ & 4.445 & 0,9 \\
\hline $\begin{array}{c}\text { Células escamosas indeterminadas de } \\
\text { alto grau }\end{array}$ & 1.010 & 0,2 \\
\hline $\begin{array}{l}\text { Células glandulares indeterminadas } \\
\text { não neoplásicas }\end{array}$ & 550 & 0,1 \\
\hline $\begin{array}{c}\text { Células glandulares indeterminadas de } \\
\text { alto grau }\end{array}$ & 162 & 0,03 \\
\hline Origem indefinida não neoplásica & 16 & 0,003 \\
\hline Origem indefinida de alto grau & 24 & 0,005 \\
\hline Lesão intraepitelial de baixo grau & 2750 & 0,5 \\
\hline Lesão intraepitelial de alto grau & 860 & 0,2 \\
\hline Lesão intraepitelial de micro invasão & 88 & 0,02 \\
\hline Carcinoma epidermóide invasor & 89 & 0,02 \\
\hline Adenocarcinoma in situ & 07 & 0,001 \\
\hline Adenocarcinoma invasor & 11 & 0,002 \\
\hline Outras neoplasias & 02 & 0,0004 \\
\hline
\end{tabular}

Fonte: Ministério da Saúde/SISCOLO- Sistema de Informação do câncer do colo do útero
DISCUSSÃO

$\mathrm{O}$ presente estudo propiciou conhecer o rastreamento do câncer de colo uterino do estado do Piauí, no período referente aos anos de 2012 a 2014. Deve-se ressaltar como limitações encontradas para a elaboração do mesmo, a utilização de dados preexistentes, disponibilizados em sistemas de informações. Mediante a isso, a análise dos dados, teve como enfoque a descrição dos casos, que futuramente, podem ser alvos de desenvolvimento de ações de proteção, assistência, investigação, prevenção e futuros estudos acerca do assunto.

De 2012 a 2014, observaram-se, de acordo com o registro do SISCOLO, 513.910 citologias realizadas por mulheres do estado do Piauí. Com destaque para o ano de 2013, no qual foram realizadas $201.318(39,2 \%)$ citologias. Esse número de citologia, encontrou-se abaixo do encontrado por Damacena et al. ${ }^{11}$, ao analisarem o período de 2006 a 2013, encontraram cerca de 604.331 citologias de mulheres da capital do Estado do Piaui. Ainda nesse estudo, os autores atribuem a redução da realização de citologias a fatores relacionados ao monitoramento inadequado dos dados do sistema analisado, mesmo que em sua gênese, o mesmo tenha sido criado para o registro total desses exames.

No presente estudo, a faixa etária que se destacou na realização da citologia, foi a de 25 a 64 anos, sendo $77,2 \%$, em contrapartida, a de menor realização, foi a de maiores de 64 anos, com 5,3\%. Esse achado é ratificado por Corrêa et al. ${ }^{12}$, ao qual o percentual verificado foi de $75,2 \%$ para a faixa etária de 25 a 59 anos.

Mediante ao percentual considerável de realização do exame em mulheres com menos de 25 anos $(17,5 \%)$, tem-se que Silva et al. ${ }^{13}$ discorrem sobre o fato de que nessa fase tem-se o início de relações sexuais, que consequentemente, aumenta a probabilidade de desenvolver o câncer cervical, tendo em vista, as alterações proliferativas do epitélio cervical, tornando-se mais suscetível a mudanças induzidas pelo HPV.

Em estudo sobre o perfil de pacientes com câncer de colo do útero foi observado maior prevalência de pacientes de raça/cor parda $(47,9 \%)$, seguida por branca $(34,4 \%)$ e preta $(5,8 \%){ }^{14}$ Resultados que corroboram com os obtidos no presente estudo. Foi observado também que a maioria $(85,6 \%)$ dos dados de raça/cor foram classificados como não informados.

Soares et al. ${ }^{15}$, aponta em seu estudo que a escolaridade se destaca como fator de risco para o desenvolvimento de câncer de colo uterino, tendo em vista, que em seus resultados, $45 \%$ das mulheres tinham ensino fundamental incompleto e 5\% eram analfabetas. De forma semelhante, Albuquerque et al. ${ }^{16}$, obteve que mulheres com ensino fundamental completo, em sua maioria ( $71 \%$ ) realizam o exame de 
citologia, em contrapartida, o menor percentual correspondia a de mulheres com ensino fundamental incompleto.

O epitélio escamoso foi o mais representado nas amostras citopatológicas $(98,9 \%)$, seguido pelo glandular $(48,25 \%)$ e metaplásico $(13,01 \%)$. Valores superiores aos obtidos no estudo de Vasconcelos e Amalbice $^{17}$, onde apenas $37,6 \%$ tiveram a representação do epitélio escamoso, 24\% tiveram os epitélios escamoso e glandular e 7,2\% tiveram escamoso e metaplásico.

Vale ressaltar que segundo o Sistema Bethesda de $2001^{18}$, um indicador de adequação e qualidade da amostra é que ela incluía a representação dos três epitélios. Assim, o significado destes achados seria melhor avaliado se o profissional responsável pela coleta registrasse no prontuário dados relevantes como: impossibilidade de coleta da endocérvice, dificuldade em visualização do colo uterino, presença de leucorréia, histerectomia prévia, entre outros ${ }^{17}$.

Em um estudo que analisou os resultados citológicos das amostras cervicovaginais coletadas em um município do Maranhão foi identificado a frequência de: bacilos 56,8\%, $\operatorname{cocos} 42,2 \%$ e lactobacilos $35,2 \%$, aproximando-se principalmente aos achados de lactobacilos neste estudo. Corroborando para isso, a presença da grande variedade de bactérias no conteúdo cérvico-vaginal está relacionada a estes agentes que são normalmente detectados em mulheres assintomáticas, formando uma flora mista composta de cocos e bacilos, além dos lactobacilos. ${ }^{19}$

A alteração celular benigna mais observada foi inflamação $(77,3 \%)$. Resultado semelhante foi observado em estudo de rastreamento do câncer de colo do útero no Estado do Maranhão com a inflamação sendo a alteração celular benigna mais frequente $(86,3 \%)^{13}$. Para Lima et al. ${ }^{20}$ o processo inflamatório intenso prejudica a qualidade da amostra, devendo ser realizado o tratamento e a nova coleta citológica após três meses.

A atipia celular mais frequente foi a de células escamosas indeterminadas não neoplásicas, presente em 4.445 exames (0,9\%). Segundo Fernandes et al. ${ }^{21}$ essas atipias são alterações comumente descritas nos resultados dos laudos citopatológicos do colo do útero, sendo considerado um resultado controverso por ser um laudo que gera subjetividade, sendo insuficientes para definir um diagnóstico de lesão intraepitelial ou invasora. Nesses casos o Instituto Nacional de Câncer (INCA) recomenda a repetição do exame citopatológico em um intervalo de seis a 12 meses $^{22}$.

Já em estudo Laganá et al. $^{23}$ as alterações citopatológicas malignas mais frequentes foram: lesão intraepitelial de baixo grau, $73,1 \%$; lesão intraepitelial de alto grau, 3,7\% e células atípicas escamosas de significado indeterminado possivelmente não neoplásicas, $2,9 \%$.

\section{CONCLUSÃO}

Diante dos resultados, observa-se um maior número de exames realizados do ano de 2013, com a maioria desses realizados em mulheres na faixa etária de 25 a 64, pardas e com ensino fundamental incompleto. $\mathrm{O}$ epitélio escamoso foi $\mathrm{o}$ mais representado nas amostras citopatológicas, sendo os agentes microbiológicos mais frequentes no colo uterino os cocos, os Lactobacillus sp e os Bacilos. Dentre as alterações celulares benignas, a inflamação foi o achado mais presente e as células escamosas indeterminadas não neoplásicas foram as atipias em células escamosas mais presente.

Um fator importante a ser considerado é a grande frequência de variáveis ignoradas ou não preenchidas. Dessa forma é importante a adoção de estratégias que melhorem a coleta desses dados, que são importantes fontes de informações em saúde e que podem ser utilizados para a implementação de possíveis políticas nesse campo e em pesquisas da área de saúde.

\section{REFERÊNCIAS}

1. Brasil. Ministério da Saúde (MS). Instituto Nacional de Câncer (Inca). Estimativa 2012: incidência de câncer no Brasil. Rio de Janeiro: Inca; 2012.

2. World Health Organization. Information Centre on Human Papilloma Virus (HPV) and Cervical Cancer. Human papillomavirus and related cancers in Brazil. Disponível em: <www.who.int/hpvcentre>. Acesso em: 14 abril. 2018.

3. Brasil. Ministério da Saúde (MS). Instituto Nacional de Câncer (Inca). Estimativa 2018: incidência de câncer no Brasil. Rio de Janeiro: Inca; 2018.

4. Santos RS, Melo ECP, Santos KM. Análise espacial dos indicadores pactuados para o rastreamento do câncer do colo do útero no Brasil. Texto contexto - enferm. 2012; 21(4):800-10.

5. Brasil. Ministério da Saúde (MS). Instituto Nacional de Câncer (Inca). Falando sobre câncer do colo do útero. Rio de Janeiro: Inca; 2002.

6. World Health Organization. National cancer control programmes: policies and managerial guidelines. 2.ed. Geneva: WHO, 2002

7. Silva RCF, Hortale VA. Cuidados paliativos oncológicos: elementos para o debate de diretrizes na área. Cad Saúde Pública. 2010; 22(10):2055-66.

8. Brasil. Ministério da Saúde (MS). Instituto Nacional de Câncer (INCA). Diretrizes brasileiras para o rastreamento do câncer do colo do útero. Rio de Janeiro: INCA; 2011. 
9. Brasil. Ministério da Saúde. Secretaria de Atenção à Saúde. Departamento de Atenção Básica. Controle dos cânceres do colo do útero e da mama. 2. ed. - Brasília: Editora do Ministério da Saúde, 2013. 124 p.

10.Brasil. Ministério da Saúde. Conselho Nacional de Saúde. Resolução No 466 de 12 de dezembro de 2012. Diário Oficial da União da República Federativa do Brasil, junho de 2013.

11.Damacena AM, Luz LL, Mattos IE. Rastreamento do câncer do colo do útero em Teresina, Piauí: estudo avaliativo dos dados do Sistema de Informação do Câncer do Colo do Útero, 20062013. Epidemiol Serv Saúde. 2017; 26(1):71-80.

12. Correa CSL, Lima AS, Leite ICG, Pereira LC, Nogueira MC, Duarte DAP et al. Rastreamento do câncer do colo do útero em Minas Gerais: avaliação a partir de dados do Sistema de Informação do Câncer do Colo do Útero (SISCOLO). Cad saúde colet. 2017; 25(3):315-23.

13.Silva DMS, Silva AMN, Brito MLO, Gomes SRL, Nascimento MSB, Chein MBC. Rastreamento do câncer do colo do útero no Estado do Maranhão, Brasil. Ciênc saúde coletiva. 2014; 19(4):1163-70 .

14. Thuler LCS, Bergmann A, Casado L. Perfil das pacientes com câncer do colo do útero no Brasil, 2000-2009: estudo de base secundária. Rev bras cancerol. 2012; 58(3):351-57.

15. Soares MC, Mishima SM, Meincke SMK, Simino GPR. Câncer de colo uterino: caracterização das mulheres em um município do sul do Brasil. Esc Anna Nery. 2010; 14(1):90-6.

16. Albuquerque KM, Frias PG, Andrade CLT, Aquino EML, Menezes G, Szwarcwald CL. Cobertura do teste de Papanicolaou e fatores associados a não-realização: um olhar sobre o Programa de Prevenção do Câncer do Colo do Útero em Pernambuco, Brasil. Cad. Saúde Pública. 2009; 25(2):301-9.

17.Vasconcelos FGA, Amabilce J. Análise da representação dos epitélios de colpocitologias oncóticas em unidade básica de saúde do município de São Paulo. Rev APS. 2007; 10(2):116-19.

18.Berek JS. Simplification of the new Bethesda 2001 classification system. Am J Obstet Gynecol. 2003; 188(3 Suppl):S2-5

19. Campos RS, Silva IC, Silva LRL, Pinto MLS, Mochel EG. Prevenção do câncer de colo uterino: achados citológicos e microbiológicos em mulheres e uma cidade do Nordeste - Maranhão. Rev Ciênc Saúde. 2010; 12(1):45-8

20.Lima TM, Castelo ARP, Oliveira RG, Costa LQ, Tavares MC, Santiago JMV et al. Analysis of the cytopathological reports from a natural childbirth center in Fortaleza-CE: A descriptive study. Online Braz J Nurs. 2009; 8(2).
21.Fernandes F, Furtado Y, Russomano F, Silva KS, Silveira R, Faria $\mathrm{P}$ et al. Diagnóstico Citopatológico de ASC-US e ASC-H no Serviço Integrado Tecnológico em Citologia do INCA. Rev bras cancerol. 2012; 58(3):453-59.

22.Brasil. Instituto Nacional de Câncer José Alencar Gomes da Silva. Coordenação de Prevenção e Vigilância. Divisão de Detecção Precoce e Apoio à Organização de Rede. Diretrizes brasileiras para o rastreamento do câncer do colo do útero. 2. ed. rev. atual. Rio de Janeiro: INCA, 2016.

23.Laganá MTC, Silva MMP, Lima LF, França TLB. Alterações Citopatológicas, Doenças sexualmente transmissíveis e periodicidade dos exames de rastreamento em Unidade Básica de Saúde. Rev bras cancerol. 2013; 59(4):523-30.

\section{CONFLITO DE INTERESSES}

Os autores declaram não haver conflitos de interesse.

\section{AUTOR PARA CORRESPONDENCIA}

\section{Gleyson Moura dos Santos}

g_leyson_moura@hotmail.com

Submetido em 05/08/2018 Aceito em 04/10/2018 